Bull. Korean Math. Soc. 49 (2012), No. 6, pp. 1311-1326

http://dx.doi.org/10.4134/BKMS.2012.49.6.1311

\title{
MULTIPLICITY OF NONTRIVIAL SOLUTIONS TO PERTURBED SCHRÖDINGER SYSTEM WITH MAGNETIC FIELDS
}

\author{
Huixing Zhang and Wenbin LiU
}

\begin{abstract}
We are concerned with the multiplicity of semiclassical solutions of the following Schrödinger system involving critical nonlinearity and magnetic fields$$
\left\{\begin{array}{l}
-(\varepsilon \nabla+i A(x))^{2} u+V(x) u=H_{u}(u, v)+K(x)|u|^{2^{*}-2} u, x \in \mathbb{R}^{N} \\
-(\varepsilon \nabla+i B(x))^{2} v+V(x) v=H_{v}(u, v)+K(x)|v|^{2}-2 v, x \in \mathbb{R}^{N},
\end{array}\right.
$$

where $2^{*}=2 N /(N-2)$ is the Sobolev critical exponent and $i$ is the imaginary unit. Under proper conditions, we prove the existence and multiplicity of the nontrivial solutions to the perturbed system.
\end{abstract}

\section{Introduction}

This paper is motivated by some works that have appeared in recent years concerning the nonlinear Schrödinger equation with critical nonlinearity and magnetic fields of the form

(1.1) $i \hbar \frac{\partial \psi}{\partial t}=-\frac{\hbar^{2}}{2 m}(\nabla+i A(x))^{2} \psi+W(x) \psi-K(x)|\psi|^{2^{*}-2} \psi-h\left(x,|\psi|^{2}\right) \psi$,

where $\hbar$ is Planck's constant, $i$ is the imaginary unit, $2^{*}$ is the critical exponent, $2^{*}=\frac{2 N}{N-2}$, for $N \geq 3, A(x)=\left(A_{1}(x), A_{2}(x), \ldots, A_{N}(x)\right): \mathbb{R}^{N} \rightarrow \mathbb{R}^{N}$ is a real vector potential with magnetic field $B=\operatorname{curl} A$ and $W(x)$ is a scalar electric potential. Knowledge of the solutions for the elliptic equation

(1.2) $-(\nabla+i A(x))^{2} u(x)+\lambda(W(x)-E) u(x)=\lambda K(x)|u|^{2^{*}-2} u+\lambda h\left(x,|u|^{2}\right) u$

has a great importance in the study of the standing-wave solutions of (1.2), i.e., the solutions of the type

$$
\psi(x, t)=\exp \left(-\frac{i E t}{\hbar}\right) u(x),
$$

Received July 17, 2011.

2010 Mathematics Subject Classification. 35B33, 35J60, 35J65.

Key words and phrases. perturbed Schrödinger system, critical nonlinearity, variational methods, magnetic fields.

This research is supported by the National Natural Science Foundation of China (11271364) and the Fundamental Research Funds for the Central Universities (2012QNA46). 
where $\lambda^{-1}=\frac{\hbar^{2}}{2 m}$.

The transition from quantum mechanics to classical mechanics can be conducted by making $\hbar \rightarrow 0$. Therefore, the existence and multiplicity of solutions for $\hbar$ small have important physical interest.

The equation in the case $A(x) \equiv 0$ has been explored by many authors including M. Del. Pino and P. Felmer [16, 17], A. Floer and A. Weinstein [14], Y. G. Oh [14] and F. Wang [20]. For much more results, we refer the reader to $[1,2,8,9,11]$ and the reference therein.

As far as the equation (1.2) in the case of $A(x) \neq 0$ is concerned, we recall P. L. Lions [13], G. Arioli and A. Szulkin [3], S. Cingolani [7] and the works of $[4,6,10,18,19,21]$.

Among the works mentioned above, the corresponding authors have obtained many valuable results. However, many results have only been established in subcritical case by using various methods.

Motivated by the results just described, a natural question is whether the existence and multiplicity of results occur for the following perturbed Schrödinger system with critical nonlinearity and magnetic fields as follows

$$
\left\{\begin{aligned}
-(\varepsilon \nabla+i A(x))^{2} u+V(x) u & =H_{u}(u, v)+K(x)|u|^{2^{*}-2} u, x \in \mathbb{R}^{N}, \\
-(\varepsilon \nabla+i B(x))^{2} v+V(x) v & =H_{v}(u, v)+K(x)|v|^{2^{*}-2} v, x \in \mathbb{R}^{N} .
\end{aligned}\right.
$$

To my knowledge, there seems some work on the existence of solutions to (1.2), but to the system (1.3), there is few work on the existence and multiplicity of solutions.

By using the similar ideas of $[12,22]$, we will establish the two main results to $(1.3)$.

Firstly, we make the following assumptions throughout the paper.

$\left(V_{0}\right) V \in C\left(\mathbb{R}^{N}, \mathbb{R}\right), V(0)=\inf _{x \in \mathbb{R}^{N}} V(x)=0$ and there is a constant $b>0$ such that the set $\nu^{b}=\left\{x \in \mathbb{R}^{N}: V(x)<b\right\}$ has finite Lebesgue measure;

$\left(A_{0}\right) A(x), B(x) \in C\left(\mathbb{R}^{N}, \mathbb{R}^{N}\right), A(0)=B(0)=0 ;$

$\left(K_{0}\right) K(x) \in C\left(\mathbb{R}^{N}\right), 0<\inf K \leq \sup K<\infty$;

$\left(H_{1}\right) H \in C^{1}\left(\mathbb{R}^{+} \times \mathbb{R}^{+}, \mathbb{R}\right), H_{s}(s, t)=o(|s|+|t|)$ and $H_{t}(s, t)=o(|s|+|t|)$;

$\left(H_{2}\right) \lim _{|s|+|t| \rightarrow \infty} \frac{H_{s}(s, t)+H_{t}(s, t)}{|s|^{\alpha}+|t|^{\beta}}=0$ for some $2<\alpha, \beta<2^{\star}-1$;

$\left(H_{3}\right)$ There exist $a_{0}>0, p, q>2, \theta \in\left(2,2^{\star}\right)$ such that $H(s, t) \geq a_{0}\left(|s|^{p}+\right.$ $\left.|t|^{q}\right)$ and $0<\theta H(s, t) \leq s H_{s}(s, t)+t H_{t}(s, t)$ for all $s>0, t>0$.

Next, we show the two main results.

Theorem 1. Assume that $\left(V_{0}\right),\left(A_{0}\right),\left(K_{0}\right),\left(H_{1}\right)-\left(H_{3}\right)$ hold. Then, for any $\sigma>0$, there exists $\varepsilon_{\sigma}>0$, such that $\varepsilon \leq \varepsilon_{\sigma}$, the perturbed Schrödinger system (1.3) has one least energy solution $\left(u_{\varepsilon}, v_{\varepsilon}\right)$ satisfying

$$
\frac{\theta-2}{2 \theta} \int_{\mathbb{R}^{N}} \varepsilon^{2}\left(\left.|\nabla| u_{\varepsilon}\right|^{2}+\left.|\nabla| v_{\varepsilon}\right|^{2}\right)+V(x)\left(\left|u_{\varepsilon}\right|^{2}+\left|v_{\varepsilon}\right|^{2}\right) \leq \sigma \varepsilon^{N} .
$$

Theorem 2. Let $\left(V_{0}\right),\left(A_{0}\right),\left(K_{0}\right)$ and $\left(H_{1}\right)-\left(H_{3}\right)$ be satisfied. Moreover, assume that $H(u, v)$ is even in $(u, v)$, then, for any $m \in N$ and $\sigma>0$, there is 
$\Lambda_{m \sigma}>0$, such that the system (1.3) has at least $m$ pairs of solutions $\left(u_{\varepsilon}, v_{\varepsilon}\right)$ which satisfy the estimate (1.4) whenever $\lambda \geq \Lambda_{m \sigma}$.

The two results above mentioned are complement for the ones in [12]. Observe that though the method used in this paper is similar to the one of [12], the procedure of the main results is not trivial. We must face our problem with complex-valued functions, at the same time, we need much more delicate estimates for the appearance of magnetic potentials $A(x)$ and $B(x)$.

This paper is organized as follows. In Section 2, we describe some preliminaries. Section 3 contains the behavior of (PS) sequences and technical Lemmas. Section 4 includes the proofs of the main results.

\section{Preliminaries}

Let $\lambda=\varepsilon^{-2}$, we think about the following equivalent system

$$
\left\{\begin{aligned}
-(\nabla+i \sqrt{\lambda} A(x))^{2} u+\lambda V(x) u & =\lambda H_{u}(u, v)+\lambda K(x)|u|^{2^{*}-2} u, x \in \mathbb{R}^{N}, \\
-(\nabla+i \sqrt{\lambda} B(x))^{2} v+\lambda V(x) v & =\lambda H_{v}(u, v)+\lambda K(x)|v|^{2^{*}-2} v, x \in \mathbb{R}^{N} .
\end{aligned}\right.
$$

In order to prove Theorem 1 , we need only prove the following result.

Theorem 3. Assume that $\left(V_{0}\right),\left(A_{0}\right),\left(K_{0}\right),\left(H_{1}\right)-\left(H_{3}\right)$ hold. Then, for $\sigma>0$, there exists $\Lambda_{\sigma}>0$ such that if $\lambda \geq \Lambda_{\sigma}$, the system (2.1) has at least one least energy solution $\left(u_{\lambda}, v_{\lambda}\right)$ which satisfies

$$
\frac{\theta-2}{2 \theta} \int_{\mathbb{R}^{N}}\left(|\nabla| u_{\lambda}||^{2}+|\nabla| v_{\lambda}||^{2}+\lambda V(x)\left(\left|u_{\lambda}\right|^{2}+\left|v_{\lambda}\right|^{2}\right) \leq \sigma \lambda^{1-\frac{N}{2}} .\right.
$$

Similarly, if we can prove the multiplicity of nontrivial solutions to $(2.1)$, Theorem 2 will be obtained.

For the convenience, we quote the necessary notations.

Let $\nabla_{A} u=(\nabla+i \sqrt{\lambda} A) u, \nabla_{B} v=(\nabla+i \sqrt{\lambda} B) v, E_{\lambda, A}\left(\mathbb{R}^{N}\right)=\left\{u \in L^{2}\left(\mathbb{R}^{N}\right)\right.$ : $\left.\nabla_{A} u \in L^{2}\left(\mathbb{R}^{N}\right)\right\}$ and $E_{\lambda, B}\left(\mathbb{R}^{N}\right)=\left\{v \in L^{2}\left(\mathbb{R}^{N}\right): \nabla_{B} v \in L^{2}\left(\mathbb{R}^{N}\right)\right\}$. It is obvious that $E_{\lambda, A}$ is the Hilbert subspace under the scalar product

$$
(u, v)_{\lambda, A}=\operatorname{Re} \int_{\mathbb{R}^{N}}\left(\left(\nabla_{A} u, \overline{\nabla_{A}} v\right)+\lambda V(x) u \bar{v}\right)
$$

the norm induced by the product $(\cdot, \cdot)$ is

$$
\|u\|_{\lambda, A}^{2}=\int_{\mathbb{R}^{N}}\left(\left|\nabla_{A} u\right|^{2}+\lambda V(x)|u|^{2}\right) .
$$

It is easily known that $E_{\lambda, A}$ is the closure of $C_{0}^{\infty}\left(\mathbb{R}^{N}, \mathbb{C}\right)$. For $E_{\lambda, B}$, there exists the similar results to $E_{\lambda, A}$.

Remark 2.1. We have the following diamagnetic inequality (see [13]):

$$
\begin{aligned}
& \left|\nabla_{A} u(x)\right| \geq|\nabla| u(x)||, u \in E_{\lambda, A}\left(\mathbb{R}^{N}\right), \\
& \left|\nabla_{B} v(x)\right| \geq|\nabla| v(x)||, v \in E_{\lambda, B}\left(\mathbb{R}^{N}\right) .
\end{aligned}
$$


Indeed, since $A, B$ is real-valued, we have

$|\nabla| u(x)||=\left|\operatorname{Re}\left(\nabla u \frac{\bar{u}}{|u|}\right)\right|=\left|\operatorname{Re}(\nabla u+i \sqrt{\lambda} A u) \frac{\bar{u}}{|u|}\right| \leq|\nabla u+i \sqrt{\lambda} A u|=\left|\nabla_{A} u(x)\right|$

and

$|\nabla| v(x)||=\left|\operatorname{Re}\left(\nabla v \frac{\bar{v}}{|v|}\right)\right|=\left|\operatorname{Re}(\nabla v+i \sqrt{\lambda} B v) \frac{\bar{v}}{|v|}\right| \leq|\nabla v+i \sqrt{\lambda} B v|=\left|\nabla_{B} v(x)\right|$

(the bar denotes complex conjugation). These facts mean if $u \in E_{\lambda, A}\left(\mathbb{R}^{N}\right)$, $v \in E_{\lambda, B}\left(\mathbb{R}^{N}\right)$, then $|u|,|v| \in H^{1}\left(\mathbb{R}^{N}\right)$ and therefore $u, v \in L^{p}\left(\mathbb{R}^{N}\right)$ for any $p \in\left[2,2^{*}\right)$, i.e., if $u_{n} \rightarrow u$ in $E_{\lambda, A}\left(v_{n} \rightarrow v\right.$ in $\left.E_{\lambda, B}\right)$, then $u_{n} \rightarrow u$ in $L_{l o c}^{p}\left(\mathbb{R}^{N}\right)$ for any $p \in\left[2,2^{*}\right)\left(v_{n} \rightarrow v\right.$ in $\left.L_{l o c}^{p}\left(\mathbb{R}^{N}\right)\right)$ and $u_{n} \rightarrow u$ a.e. in $\mathbb{R}^{N}\left(v_{n} \rightarrow v\right.$ a.e. in $\left.\mathbb{R}^{N}\right)$.

Remark 2.2. In general, $E_{\lambda, A}\left(\mathbb{R}^{N}\right) \nsubseteq H^{1}\left(\mathbb{R}^{N}\right)$ and $H^{1}\left(\mathbb{R}^{N}\right) \nsubseteq E_{\lambda, A}\left(\mathbb{R}^{N}\right)$. However, it is proved by Szulkin that if $\Omega$ is a bounded domain with regular boundary, then, the spaces $E_{\lambda, A}(\Omega)$ and $H^{1}(\Omega)$ are equivalent, where $E_{\lambda, A}(\Omega)=\left\{u \in L^{2}(\Omega): \nabla_{A} u \in L^{2}(\Omega)\right\}$ with the norm

$$
\|u\|_{E_{\lambda, A}(\Omega)}^{2}=\int_{\Omega}\left(\left|\nabla_{A} u\right|^{2}+|u|^{2}\right)
$$

From Remark 2.1, for each $s \in\left[2,2^{*}\right.$ ), there is $c_{s}>0$ (independent of $\lambda$ ) such that, if $\lambda>1$, we have

$$
\left(\int_{\mathbb{R}^{N}}|u|^{s}\right)^{\frac{1}{s}} \leq c_{s}\left(\left.\int_{\mathbb{R}^{N}}|\nabla| u\right|^{2}\right)^{\frac{1}{2}} \leq c_{s}\left(\int_{\mathbb{R}^{N}}\left|\nabla_{A} u\right|^{2}\right)^{\frac{1}{2}} \leq c_{s}\|u\|_{E_{\lambda, A}} .
$$

Set $E_{\lambda}=E_{\lambda, A} \times E_{\lambda, B}$ and $\|(u, v)\|_{\lambda}^{2}=\|u\|_{\lambda, A}^{2}+\|v\|_{\lambda, B}^{2}$ for $(u, v) \in E_{\lambda}$. The energy functional associated with (2.1) is defined by

$$
\begin{aligned}
J_{\lambda}(u, v)= & \frac{1}{2} \int_{\mathbb{R}^{N}}\left(|\nabla u+i \sqrt{\lambda} A u|^{2}+|\nabla v+i \sqrt{\lambda} B v|^{2}\right. \\
& \left.+\lambda V(x)\left(|u|^{2}+|v|^{2}\right)\right)-\lambda \int_{\mathbb{R}^{N}} G(x, u, v) \\
= & \frac{1}{2}\|(u, v)\|_{\lambda}^{2}-\lambda \int_{\mathbb{R}^{N}} G(x, u, v) \quad \text { for }(u, v) \in E_{\lambda},
\end{aligned}
$$

where $G(x, u, v)=\frac{K(x)}{2^{*}}\left(|u|^{2^{*}}+|v|^{2^{*}}\right)+H(u, v)$.

Under the assumptions of Theorem 1, standard arguments [22] indicate that $J_{\lambda} \in C^{1}\left(E_{\lambda}, \mathbb{R}\right)$ and the critical points of $J_{\lambda}$ are weak solutions of $(2.1)$.

Moreover, for convenience, let us recall the definition and some properties of the Krasnoselski genus [5].

The concept of the index theory is most easily explained for an even functional $E$ on some Banach space $X$ with symmetry group $G=Z_{2}=\{i d,-i d\}$.

Define

$$
\mathbb{A}=\{A \in X \mid A \text { is closed and } A=-A\}
$$

to be the class of closed symmetric subsets of $X$. 
Definition 2.1. For $A \in \mathbb{A}$, define the Krasnoselskii genus of $A$, denoted by $i(A)$, as

$$
i(A)=\left\{\begin{array}{l}
0, \quad A=\phi, \\
\inf \left\{m ; \exists h \in C^{0}\left(A, \mathbb{R}^{m} /\{0\}\right), h(-u)=-h(u)\right\}, \\
\infty, \text { if }\left\{m ; \exists h \in C^{0}\left(A, \mathbb{R}^{m} /\{0\}\right), h(-u)=-h(u)\right\}=\phi .
\end{array}\right.
$$

It is easy to see that $i(A)=\infty$, if $0 \in A$.

Moreover, the Krasnoselskii genus has the following properties.

Lemma 2.1. (1) $i(A) \geq 0, \quad i(A)=0 \Longleftrightarrow A=\emptyset$;

(2) (Normalization) $i(A)=1$ if $A$ contains only a pair symmetric points;

(3) (Monotonicity) For any $A, B \in \mathbb{A}$, if $A \sqsubset B$, then $i(A) \leq i(B)$;

(4) (Subadditivity) $i(A \cup B) \leq i(A)+i(B), \quad \forall A, B \in \mathbb{A}$;

(5) (Super-variant) For any continuous odd $\operatorname{map} \varphi: X \rightarrow X$ and set $A \in \mathbb{A}$, there holds $i(A) \leq i(\overline{\varphi A})$;

(6) (Continuity) For any $A \in \mathbb{A}$, if $A$ is compact, there exists a symmetric neighborhood $N$ of $A$ such that $i(\bar{N})=i(A)$; Furthermore, if $A$ is compact and $0 \in A$, then $i(A)<\infty$;

(7) Suppose $X_{1}$ is a m-dimensional subspace of $X$ and $S$ is the surface of unit ball in $X$, then $i\left(X_{1} \cap S\right)=m$;

(8) Suppose $X=X_{1} \oplus X_{2}, \operatorname{dim} X_{1}=m$, if $A, B \in \mathbb{A}$ and $i(A)>m$, then $A \cap X_{2} \neq \emptyset$.

\section{Technical lemmas}

We call a sequence $\left\{\left(u_{n}, v_{n}\right)\right\} \subset E_{\lambda}$ is a $(P S)_{c}$ sequence, if

$$
J_{\lambda}\left(u_{n}, v_{n}\right) \rightarrow c, J_{\lambda}^{\prime}\left(u_{n}, v_{n}\right) \rightarrow 0 \text { in } E_{\lambda}^{-1} .
$$

$J_{\lambda}$ is said to satisfy the $(P S)_{c}$ condition, if any $(P S)_{c}$ sequence contains a convergent subsequence.

Similar to the proof of Lemma 3.1 in [12], the following result can be obtained.

Lemma 3.1. Assume that the assumptions of Theorem 3 hold and $\left\{\left(u_{n}, v_{n}\right)\right\}$ is a $(P S)_{c}$ sequence for $J_{\lambda}$. Then, $c \geq 0$ and $\left\{\left(u_{n}, v_{n}\right)\right\}$ is bounded in $E_{\lambda}$.

Proof. By $\left(H_{3}\right)$, we have

$$
\begin{aligned}
& J_{\lambda}\left(u_{n}, v_{n}\right)-\frac{1}{\theta} J_{\lambda}^{\prime}\left(u_{n}, v_{n}\right)\left(u_{n}, v_{n}\right) \\
= & \left(\frac{1}{2}-\frac{1}{\theta}\right)\left\|\left(u_{n}, v_{n}\right)\right\|_{\lambda}^{2}+\left(\frac{1}{\theta}-\frac{1}{2^{*}}\right) \lambda \int_{\mathbb{R}^{N}} K(x)\left(\left|u_{n}\right|^{2^{*}}+\left|v_{n}\right|^{2^{*}}\right) \\
& +\lambda \int_{\mathbb{R}^{N}}\left(\frac{1}{\theta}\left(u_{n} H_{u}\left(u_{n}, v_{n}\right)+v_{n} H_{v}\left(u_{n}, v_{n}\right)\right)-H\left(u_{n}, v_{n}\right)\right) \\
\geq & \left(\frac{1}{2}-\frac{1}{\theta}\right)\left\|\left(u_{n}, v_{n}\right)\right\|_{\lambda}^{2} \geq 0 .
\end{aligned}
$$

So $\left\{\left(u_{n}, v_{n}\right)\right\}$ is bounded in $E_{\lambda}$ and $c \geq 0$. The proof is completed. 
By Lemma 3.1, $(P S)_{c}$ sequence $\left\{\left(u_{n}, v_{n}\right)\right\}$ is bounded in $E_{\lambda}$. So we can assume $\left(u_{n}, v_{n}\right) \rightarrow(u, v)$ in $E_{\lambda}$. By Remark 2.1, passing to a subsequence, $u_{n} \rightarrow u, v_{n} \rightarrow v$ in $L_{l o c}^{p}\left(\mathbb{R}^{N}\right)$ for any $p \in\left[2,2^{*}\right)$ and $u_{n} \rightarrow u, v_{n} \rightarrow v$ a.e. in $\mathbb{R}^{N}$. It is standard that $(u, v)$ is a critical point of $J_{\lambda}$, namely a weak solution of $(2.1)$.

Lemma 3.2. Let $s \in\left[2,2^{*}\right)$. Then there is a subsequence $\left\{\left(u_{n_{j}}, v_{n_{j}}\right)\right\}$ such that for any $\varepsilon>0$, there exists $r_{\varepsilon}>0$ with

$$
\limsup _{i \rightarrow \infty} \int_{B_{i} \backslash B_{r}}\left|u_{n_{i}}\right|^{s}+\left|v_{n_{i}}\right|^{s} \leq \epsilon \quad \text { for all } r \geq r_{\epsilon},
$$

where $B_{r}:=\left\{x \in \mathbb{R}^{N}:|x| \leq r\right\}$.

Proof. From $u_{n} \rightarrow u$ and $v_{n} \rightarrow v$ in $L_{l o c}^{s}\left(\mathbb{R}^{N}\right)$, we have

$$
\int_{B_{i}}\left|u_{n}\right|^{s}+\left|v_{n}\right|^{s} \rightarrow \int_{B_{i}}|u|^{s}+|v|^{s} \quad \text { as } n \rightarrow \infty .
$$

Thus, there exists $\tilde{n}_{i} \in \mathbb{N}$ such that

$$
\int_{B_{i}}\left(\left|u_{n_{j}}\right|^{s}-|u|^{s}\right)+\left(\left|v_{n_{j}}\right|^{s}-|v|^{s}\right)<\frac{1}{i} \quad \text { for all } n_{j}=\tilde{n}_{i}+j, j=1,2, \ldots
$$

Let $\tilde{n}_{i+1} \geq \tilde{n}_{i}$ and for $n_{i}=\tilde{n}_{i}+i$, we have

$$
\int_{B_{i}}\left(\left|u_{n_{i}}\right|^{s}-|u|^{s}\right)+\left(\left|v_{n_{i}}\right|^{s}-|v|^{s}\right)<\frac{1}{i} \quad \text { for all } n_{i}=\tilde{n}_{i}+i, i=1,2, \ldots
$$

Observe that there exists $r_{\epsilon}>0$ satisfying

$$
\int_{\mathbb{R}^{N} \backslash B_{r}}|u|^{s}+|v|^{s}<\frac{\varepsilon}{3} \quad \text { for all } r \geq r_{\varepsilon} .
$$

Therefore,

$$
\begin{aligned}
\int_{B_{i} \backslash B_{r}}\left|u_{n_{i}}\right|^{s}+\left|v_{n_{i}}\right|^{s} & =\int_{B_{i}}\left|u_{n_{i}}\right|^{s}-|u|^{s}+\int_{B_{i} \backslash B_{r}}|u|^{s}+\int_{B_{r}}|u|^{s}-\left|u_{n_{i}}\right|^{s} \\
& \leq \frac{1}{i}+\int_{\mathbb{R}^{N} \backslash B_{r}}|u|^{s}+\int_{B_{r}}|u|^{s}-\left|u_{n_{i}}\right|^{s} \\
& \leq \varepsilon \quad \text { as } i \rightarrow \infty .
\end{aligned}
$$

The proof is completed.

Let $\eta \in C^{\infty}\left(\mathbb{R}^{+}\right)$which satisfy $0 \leq \eta(t) \leq 1, t \geq 0$. $\eta(t)=1$, if $t \leq 1$ and $\eta(t)=0$, if $t \geq 2$. Define $\widetilde{u}_{j}(x)=\eta(2|x| / j) u(x), \widetilde{v}_{j}(x)=\eta(2|x| / j) v(x)$, then $\widetilde{u}_{j} \rightarrow u$ in $E_{1, A}$ and $\widetilde{v}_{j} \rightarrow v$ in $E_{1, B}$.

\section{Lemma 3.3.}

$(3.1) \lim _{j \rightarrow \infty}\left|\int_{\mathbb{R}^{N}}\left(H_{u}\left(u_{n_{j}}, v_{n_{j}}\right)-H_{u}\left(u_{n_{j}}-\widetilde{u}_{j}, v_{n_{j}}-\widetilde{v}_{j}\right)-H_{u}\left(\widetilde{u}_{j}, \widetilde{v}_{j}\right)\right) \varphi\right|=0$,
$(3.2) \lim _{j \rightarrow \infty}\left|\int_{\mathbb{R}^{N}}\left(H_{v}\left(u_{n_{j}}, v_{n_{j}}\right)-H_{v}\left(u_{n_{j}}-\widetilde{u}_{j}, v_{n_{j}}-\widetilde{v}_{j}\right)-H_{v}\left(\widetilde{u}_{j}, \widetilde{v}_{j}\right)\right) \psi\right|=0$ 
uniformly in $(\varphi, \psi) \in E_{1}$ with $\|(\varphi, \psi)\|_{E_{1}} \leq 1$.

Proof. Note that $\tilde{u}_{j} \rightarrow u$ in $E_{1, A}$ and $u_{j} \rightarrow u$ in $E_{1, A}$, the local compactness of Sobolev embedding implies that, for any $r>0$,

$$
\lim _{j \rightarrow \infty}\left|\int_{B_{r}}\left(H_{u}\left(u_{n_{j}}, v_{n_{j}}\right)-H_{u}\left(u_{n_{j}}-\widetilde{u}_{j}, v_{n_{j}}-\widetilde{v}_{j}\right)-H_{u}\left(\widetilde{u}_{j}, \widetilde{v}_{j}\right)\right) \varphi\right|=0
$$

uniformly in $\|\varphi\|_{1, A} \leq 1$. For any $\varepsilon>0$, there exists $r_{\varepsilon}>0$ such that

$$
\limsup _{i \rightarrow \infty} \int_{B_{i} \backslash B_{r}}\left|\widetilde{u}_{i}\right|^{s} \leq \int_{\mathbb{R}^{N} \backslash B_{r}}|u|^{s} \leq \varepsilon \quad \text { for all } r \geq r_{\varepsilon} .
$$

It follows from Lemma 3.2 (for $s=2, \alpha+1, \beta+1$ ) that

$$
\begin{aligned}
& \lim _{j \rightarrow \infty}\left|\int_{\mathbb{R}^{N}}\left(H_{u}\left(u_{n_{j}}, v_{n_{j}}\right)-H_{u}\left(u_{n_{j}}-\widetilde{u}_{j}, v_{n_{j}}-\widetilde{v}_{j}\right)-H_{u}\left(\widetilde{u}_{j}, \widetilde{v}_{j}\right)\right) \varphi\right| \\
= & \lim _{j \rightarrow \infty}\left|\int_{B_{j} \backslash B_{r}}\left(H_{u}\left(u_{n_{j}}, v_{n_{j}}\right)-H_{u}\left(u_{n_{j}}-\widetilde{u}_{j}, v_{n_{j}}-\widetilde{v}_{j}\right)-H_{u}\left(\widetilde{u}_{j}, \widetilde{v}_{j}\right)\right) \varphi\right| \\
\leq & c_{1} \limsup _{j \rightarrow \infty} \int_{B_{j} \backslash B_{r}}\left(\left|u_{n_{j}}\right|+\left|v_{n_{j}}\right|+\left|\widetilde{u}_{j}\right|+\left|\widetilde{v}_{j}\right|\right)|\varphi| \\
& +c_{2} \limsup _{j \rightarrow \infty} \int_{B_{j} \backslash B_{r}}\left(\left|u_{n_{j}}\right|^{\alpha}+\left|v_{n_{j}}\right|^{\beta}+\left|\widetilde{u}_{j}\right|^{\alpha}+\left|\widetilde{v}_{j}\right|^{\beta}\right)|\varphi| \\
\leq & c_{1} \limsup _{j \rightarrow \infty}\left(\left\|u_{n_{j}}\right\|_{L^{2}\left(B_{j} \backslash B_{r}\right)}+\left\|v_{n_{j}}\right\|_{L^{2}\left(B_{j} \backslash B_{r}\right)}\right. \\
& \left.+\left\|\widetilde{u}_{j}\right\|_{L^{2}\left(B_{j} \backslash B_{r}\right)}+\left\|\widetilde{v}_{j}\right\|_{L^{2}\left(B_{j} \backslash B_{r}\right)}\right)\|\varphi\|_{L^{2}} \\
& +c_{2}\left(\limsup _{j \rightarrow \infty}\left(\left\|u_{n_{j}}\right\|_{L^{\alpha+1}\left(B_{j} \backslash B_{r}\right)}^{\alpha}+\left\|\widetilde{u}_{j}\right\|_{L^{\alpha+1}\left(B_{j} \backslash B_{r}\right)}^{\alpha}\right)\|\varphi\|_{L^{\alpha+1}}\right. \\
& \left.+\limsup _{j \rightarrow \infty}\left(\left\|v_{n_{j}}\right\|_{L^{\beta+1}\left(B_{j} \backslash B_{r}\right)}^{\beta}+\left\|\widetilde{v}_{j}\right\|_{L^{\beta+1}\left(B_{j} \backslash B_{r}\right)}^{\beta}\right)\|\varphi\|_{L^{\beta+1}}\right) \\
\leq & c_{3} \varepsilon^{\frac{1}{2}}+c_{4} \varepsilon^{\frac{\alpha}{\alpha+1}}+c_{5} \varepsilon^{\frac{\beta}{\beta+1}}
\end{aligned}
$$

which implies the conclusion is correct. Similarly, we have that

$$
\lim _{j \rightarrow \infty}\left|\int_{\mathbb{R}^{N}}\left(H_{v}\left(u_{n_{j}}, v_{n_{j}}\right)-H_{v}\left(u_{n_{j}}-\widetilde{u}_{j}, v_{n_{j}}-\widetilde{v}_{j}\right)-H_{v}\left(\widetilde{u}_{j}, \widetilde{v}_{j}\right)\right) \psi\right|=0 .
$$

The proof is completed.

Lemma 3.4. One has along a subsequence

$$
\begin{aligned}
& J_{\lambda}\left(u_{n}-\widetilde{u}_{n}, v_{n}-\widetilde{v}_{n}\right) \rightarrow c-J_{\lambda}(u, v), \\
& J_{\lambda}^{\prime}\left(u_{n}-\widetilde{u}_{n}, v_{n}-\widetilde{v}_{n}\right) \rightarrow 0 \text { in } E_{\lambda}^{-1} .
\end{aligned}
$$

Proof. Since $\widetilde{u}_{j} \rightarrow u$ in $E_{1, A}, \widetilde{v}_{j} \rightarrow v$ in $E_{1, B}$ and $\left(u_{j}, v_{j}\right) \rightarrow(u, v)$ in $E_{\lambda}$, one has

$$
J_{\lambda}\left(u_{n}-\widetilde{u}_{n}, v_{n}-\widetilde{v}_{n}\right)
$$




$$
\begin{aligned}
= & J_{\lambda}\left(u_{n}, v_{n}\right)-J_{\lambda}\left(\widetilde{u}_{n}, \widetilde{v}_{n}\right) \\
& +\frac{\lambda}{2^{*}} \int_{\mathbb{R}^{N}} K(x)\left(\left|u_{n}\right|^{2^{*}}-\left|u_{n}-\widetilde{u}_{n}\right|^{2^{*}}-\left|\widetilde{u}_{n}\right|^{2^{*}}\right) \\
& +\frac{\lambda}{2^{*}} \int_{\mathbb{R}^{N}} K(x)\left(\left|v_{n}\right|^{2^{*}}-\left|v_{n}-\widetilde{v}_{n}\right|^{2^{*}}-\left|\widetilde{v}_{n}\right|^{2^{*}}\right) \\
& +\lambda \int_{\mathbb{R}^{N}} H\left(u_{n}, v_{n}\right)-H\left(u_{n}-v_{n}, \widetilde{u}_{n}-\widetilde{v}_{n}\right)-H\left(\widetilde{u}_{n}, \widetilde{v}_{n}\right)+o(1) .
\end{aligned}
$$

Along the lines in proving the Brezis-Lieb lemma, it is easy to check that

$$
\begin{aligned}
& \lim _{n \rightarrow \infty} \int_{\mathbb{R}^{N}} K(x)\left(\left|u_{n}\right|^{2^{*}}-\left|u_{n}-\widetilde{u}_{n}\right|^{2^{*}}-\left|\widetilde{u}_{n}\right|^{2^{*}}\right)=0, \\
& \lim _{n \rightarrow \infty} \int_{\mathbb{R}^{N}} K(x)\left(\left|v_{n}\right|^{2^{*}}-\left|v_{n}-\widetilde{u}_{n}\right|^{2^{*}}-\left|\widetilde{v}_{n}\right|^{2^{*}}\right)=0
\end{aligned}
$$

and

$$
\lim _{n \rightarrow \infty} \int_{\mathbb{R}^{N}} H\left(u_{n}, v_{n}\right)-H\left(u_{n}-v_{n}, \widetilde{u}_{n}-\widetilde{v}_{n}\right)-H\left(\widetilde{u}_{n}, \widetilde{v}_{n}\right)=0 .
$$

Note that $J_{\lambda}\left(u_{n}, v_{n}\right) \rightarrow c$ and $J_{\lambda}\left(\widetilde{u}_{n}, \widetilde{v}_{n}\right) \rightarrow J_{\lambda}(u, v)$, we get

$$
J_{\lambda}\left(u_{n}-\widetilde{u}_{n}, v_{n}-\widetilde{v}_{n}\right) \rightarrow c-J_{\lambda}(u, v) .
$$

For any $(\varphi, \psi) \in E_{\lambda}$, we have

$$
\begin{aligned}
& J_{\lambda}^{\prime}\left(u_{n}-\widetilde{u}_{n}, v_{n}-\widetilde{v}_{n}\right)(\varphi, \psi) \\
= & J_{\lambda}^{\prime}\left(u_{n}, v_{n}\right)(\varphi, \psi)-J_{\lambda}^{\prime}\left(\widetilde{u}_{n}, \widetilde{v}_{n}\right)(\varphi, \psi) \\
& +\lambda \int_{\mathbb{R}^{N}} K(x)\left(\left|u_{n}\right|^{2^{*}-2} u_{n}-\left|u_{n}-\widetilde{u}_{n}\right|^{2^{*}-2}\left(u_{n}-\widetilde{u}_{n}\right)-\left|\widetilde{u}_{n}\right|^{2^{*}-2} \widetilde{u}_{n}\right) \varphi \\
& +\lambda \int_{\mathbb{R}^{N}} K(x)\left(\left|v_{n}\right|^{2^{*}-2} v_{n}-\left|v_{n}-\widetilde{v}_{n}\right|^{2^{*}-2}\left(v_{n}-\widetilde{v}_{n}\right)-\left|\widetilde{v}_{n}\right|^{2^{*}-2} \widetilde{v}_{n}\right) \psi \\
& +\lambda \int_{\mathbb{R}^{N}}\left(H_{u}\left(u_{n}, v_{n}\right)-H_{u}\left(u_{n}-\widetilde{u}_{n}, v_{n}-\widetilde{v}_{n}\right)-H_{u}\left(\widetilde{u}_{n}, \widetilde{v}_{n}\right)\right) \varphi \\
& +\lambda \int_{\mathbb{R}^{N}}\left(H_{v}\left(u_{n}, v_{n}\right)-H_{v}\left(u_{n}-\widetilde{u}_{n}, v_{n}-\widetilde{v}_{n}\right)-H_{v}\left(\widetilde{u}_{n}, \widetilde{v}_{n}\right)\right) \psi .
\end{aligned}
$$

It is standard to check that

$$
\lim _{n \rightarrow \infty} \int_{\mathbb{R}^{N}} K(x)\left(\left|u_{n}\right|^{2^{*}-2} u_{n}-\left|u_{n}-\widetilde{u}_{n}\right|^{2^{*}-2}\left(u_{n}-\widetilde{u}_{n}\right)-\left|\widetilde{u}_{n}\right|^{2^{*}-2} \widetilde{u}_{n}\right) \varphi=0
$$

and

$$
\lim _{n \rightarrow \infty} \int_{\mathbb{R}^{N}} K(x)\left(\left|v_{n}\right|^{2^{*}-2} v_{n}-\left|v_{n}-\widetilde{v}_{n}\right|^{2^{*}-2}\left(v_{n}-\widetilde{v}_{n}\right)-\left|\widetilde{v}_{n}\right|^{2^{*}-2} \widetilde{v}_{n}\right) \psi=0
$$

uniformly in $\|(\varphi, \psi)\|_{\lambda} \leq 1$. Therefore, the conclusion required holds by Lemma 3.3. The proof is completed. 
Let $u_{n}^{1}=u_{n}-\widetilde{u}_{n}, v_{n}^{1}=v_{n}-\widetilde{v}_{n}$, then $u_{n}-u=u_{n}^{1}+\left(\widetilde{u}_{n}-u\right), v_{n}-v=$ $v_{n}^{1}+\left(\widetilde{v}_{n}-v\right)$. So $\left(u_{n}, v_{n}\right) \rightarrow(u, v)$ in $E_{\lambda}$ if and only if $\left(u_{n}^{1}, v_{n}^{1}\right) \rightarrow(0,0)$ in $E_{\lambda}$.

Note that

$$
\begin{aligned}
& J_{\lambda}\left(u_{n}^{1}, v_{n}^{1}\right)-\frac{1}{2} J_{\lambda}^{\prime}\left(u_{n}^{1}, v_{n}^{1}\right)\left(u_{n}^{1}, v_{n}^{1}\right) \\
= & \left(\frac{1}{2}-\frac{1}{2^{*}}\right) \lambda \int_{\mathbb{R}^{N}} K(x)\left(\left|u_{n}^{1}\right|^{2^{*}}+\left|v_{n}^{1}\right|^{2^{*}}\right) \\
& +\lambda \int_{\mathbb{R}^{N}}\left(\frac{1}{2}\left(u_{n}^{1} H_{u}\left(u_{n}^{1}, v_{n}^{1}\right)+v_{n}^{1} H_{v}\left(u_{n}^{1}, v_{n}^{1}\right)\right)-H\left(u_{n}^{1}, v_{n}^{1}\right)\right) \\
\geq & \frac{\lambda}{N} K_{\min } \int_{\mathbb{R}^{N}}\left(\left|u_{n}^{1}\right|^{2^{*}}+\left|v_{n}^{1}\right|^{2^{*}}\right),
\end{aligned}
$$

where $K_{\min }=\inf _{x \in \mathbb{R}^{N}} K(x)>0$. Hence by Lemma 3.4, we get

$$
\left\|u_{n}^{1}\right\|_{2^{*}}^{2^{*}}+\left\|v_{n}^{1}\right\|_{2^{*}}^{2^{*}} \leq \frac{N\left(c-J_{\lambda}(u, v)\right)}{\lambda K_{\min }}+o(1) .
$$

Now, we determine the energy level of the functional $J_{\lambda}$ below which the $(P S)$ condition holds.

Let $V_{b}(x)=\max \{V(x), b\}$, where $b$ is the positive constant in the assumption $\left(V_{0}\right)$. Since the set $\nu^{b}$ has finite measure and $u_{n}^{1}, v_{n}^{1} \rightarrow 0$ in $L_{\text {loc }}^{2}\left(\mathbb{R}^{N}\right)$, we have

$$
\int_{\mathbb{R}^{N}} V(x)\left(\left|u_{n}^{1}\right|^{2}+\left|v_{n}^{1}\right|^{2}\right)=\int_{\mathbb{R}^{N}} V_{b}(x)\left(\left|u_{n}^{1}\right|^{2}+\left|v_{n}^{1}\right|^{2}\right)+o(1) .
$$

By $\left(H_{2}\right)$ and $\left(H_{3}\right)$, there exists $C_{b}>0$ such that

$$
\begin{aligned}
& \int_{\mathbb{R}^{N}} K(x)\left(|u|^{2^{*}}+|v|^{2^{*}}\right)+u H_{u}(u, v)+v H_{v}(u, v) \\
\leq & b\left(\|u\|_{2}^{2}+\|v\|_{2}^{2}\right)+C_{b}\left(\|u\|_{2^{*}}^{2^{*}}+\|v\|_{2^{*}}^{2^{*}}\right) .
\end{aligned}
$$

Let $S$ be the best Sobolev constant satisfying

$$
S\|u\|_{2^{*}}^{2} \leq \int_{\mathbb{R}^{N}}|\nabla u|^{2} \quad \text { for all } u \in H^{1}\left(\mathbb{R}^{N}\right) .
$$

Lemma 3.5. Under the assumptions of Theorem 3, there is a constant $\alpha_{0}>0$ (independent of $\lambda$ ) such that, for any $(P S)_{c}$ sequence $\left\{\left(u_{n}, v_{n}\right)\right\} \subset E_{\lambda}$ for $J_{\lambda}$ with $\left(u_{n}, v_{n}\right) \rightarrow(u, v)$, either $\left(u_{n}, v_{n}\right) \rightarrow(u, v)$ or $c-J_{\lambda}(u, v) \geq \alpha_{0} \lambda^{1-\frac{N}{2}}$.

Proof. Assume that $\left(u_{n}, v_{n}\right) \nrightarrow(u, v)$, we have

$$
\liminf _{n \rightarrow \infty}\left\|\left(u_{n}^{1}, v_{n}^{1}\right)\right\|_{\lambda}>0 \text { and } c-J_{\lambda}(u, v)>0 .
$$

By the Sobolev inequality, we obtain

$$
\begin{aligned}
& S\left(\left\|u_{n}^{1}\right\|_{2^{*}}^{2}+\left\|v_{n}^{1}\right\|_{2^{*}}^{2}\right) \\
\leq & \int_{\mathbb{R}^{N}}\left(|\nabla| u_{n}^{1}\left\|^{2}+|\nabla| v_{n}^{1}\right\|^{2}+\lambda V(x)\left(\left|u_{n}^{1}\right|^{2}+\left|v_{n}^{1}\right|^{2}\right)\right)-\int_{\mathbb{R}^{N}} \lambda V(x)\left(\left|u_{n}^{1}\right|^{2}+\left|v_{n}^{1}\right|^{2}\right) \\
\leq & \int_{\mathbb{R}^{N}}\left|\nabla u_{n}^{1}+i A u_{n}^{1}\right|^{2}+\left|\nabla v_{n}^{1}+i B v_{n}^{1}\right|^{2}+\lambda V(x)\left(\left|u_{n}^{1}\right|^{2}+\left|v_{n}^{1}\right|^{2}\right)
\end{aligned}
$$




$$
\begin{aligned}
& -\int_{\mathbb{R}^{N}} \lambda V(x)\left(\left|u_{n}^{1}\right|^{2}+\left|v_{n}^{1}\right|^{2}\right) \\
= & \lambda \int_{\mathbb{R}^{N}} K(x)\left(\left|u_{n}^{1}\right|^{2^{*}}+\left|v_{n}^{1}\right|^{2^{*}}\right)+u_{n}^{1} H_{u}\left(u_{n}^{1}, v_{n}^{1}\right)+v_{n}^{1} H_{v}\left(u_{n}^{1}, v_{n}^{1}\right) \\
& -\int_{\mathbb{R}^{N}} \lambda V_{b}(x)\left(\left|u_{n}^{1}\right|^{2}+\left|v_{n}^{1}\right|^{2}\right)+o(1) \\
\leq & \lambda b\left(\left\|u_{n}^{1}\right\|_{2}^{2}+\left\|v_{n}^{1}\right\|_{2}^{2}\right)+C_{b} \lambda\left(\left\|u_{n}^{1}\right\|_{2^{*}}^{2^{*}}+\left\|v_{n}^{1}\right\|_{2^{*}}^{2^{*}}\right)-\lambda b\left(\left\|u_{n}^{1}\right\|_{2}^{2}+\left\|v_{n}^{1}\right\|_{2}^{2}\right)+o(1) \\
= & \lambda C_{b}\left(\left\|u_{n}^{1}\right\|_{2^{*}}^{2^{*}}+\left\|v_{n}^{1}\right\|_{2^{*}}^{2^{*}}\right)+o(1) .
\end{aligned}
$$

Meanwhile, it is easy to show that

$$
\liminf _{n \rightarrow \infty}\left\|u_{n}^{1}\right\|_{2^{*}}^{2^{*}}+\left\|v_{n}^{1}\right\|_{2^{*}}^{2^{*}}>0 .
$$

Thus, by (3.3), we get

$$
\begin{aligned}
S & \leq \lambda C_{b}\left(\left\|u_{n}^{1}\right\|_{2^{*}}^{2^{*}}+\left\|v_{n}^{1}\right\|_{2^{*}}^{2^{*}}\right)^{\frac{2^{*}-2}{2^{*}}}+o(1) \\
& \leq \lambda C_{b}\left(\frac{N\left(c-J_{\lambda}(u, v)\right)}{\lambda K_{\min }}\right)^{\frac{2}{N}}+o(1) \\
& =\lambda^{1-\frac{2}{N}} C_{b}\left(\frac{N}{K_{\min }}\right)^{\frac{2}{N}}\left(c-J_{\lambda}(u, v)\right)^{\frac{2}{N}}+o(1) .
\end{aligned}
$$

Therefore, $\alpha_{0} \lambda^{1-\frac{N}{2}} \leq c-J_{\lambda}(u, v)+o(1)$, where $\alpha_{0}=S^{\frac{N}{2}} C_{b}^{-\frac{N}{2}} N^{-1} K_{\min }$. The proof is completed.

Since $H^{1}\left(\mathbb{R}^{N}\right) \hookrightarrow L^{2^{*}}\left(\mathbb{R}^{N}\right)$ is not compact, $J_{\lambda}$ does not satisfy the $(P S)_{c}$ condition for all $c>0$. But Lemma 3.5 implies that $J_{\lambda}$ satisfies the following local $(P S)_{c}$ condition.

Lemma 3.6. Under the assumptions of Theorem 3, there is a constant $\alpha_{0}>0$ (independent of $\lambda$ ) such that, if a sequence $\left\{\left(u_{n}, v_{n}\right)\right\} \subset E_{\lambda}$ satisfies

$$
J_{\lambda}\left(u_{n}, v_{n}\right) \rightarrow c<\alpha_{0} \lambda^{1-\frac{N}{2}} \text { and } J_{\lambda}^{\prime}\left(u_{n}, v_{n}\right) \rightarrow 0 \text { in } E_{\lambda}^{-1},
$$

then, $\left\{\left(u_{n}, v_{n}\right)\right\}$ is relatively compact in $E_{\lambda}$.

Next, we consider $\lambda \geq 1$. By the assumptions of Theorem 3, we can see that $J_{\lambda}$ has the mountain-pass structure.

Lemma 3.7. Under the assumptions of Theorem 3, there exist $\alpha_{\lambda}, \rho_{\lambda}>0$ such that

$$
J_{\lambda}(u, v)>0,0<\|(u, v)\|_{\lambda}<\rho_{\lambda} ; J_{\lambda}(u, v) \geq \alpha_{\lambda}, \text { if }\|(u, v)\|_{\lambda}=\rho_{\lambda} .
$$

Proof. By $\left(H_{1}\right)-\left(H_{3}\right)$, for $\delta \leq\left(4 \lambda C_{2}\right)^{-1}$, there exists $C_{\delta}$ such that

$$
\int_{\mathbb{R}^{N}} G(x, u, v) \leq \delta\left(\|u\|_{2}^{2}+\|v\|_{2}^{2}\right)+C_{\delta}\left(\|u\|_{2^{*}}^{2^{*}}+\|v\|_{2^{*}}^{2^{*}}\right) .
$$

Thus

$$
J_{\lambda}(u, v) \geq \frac{1}{2}\|(u, v)\|_{\lambda}^{2}-\lambda \delta\left(\|u\|_{2}^{2}+\|v\|_{2}^{2}\right)-\lambda C_{\delta}\left(\|u\|_{2^{*}}^{2^{*}}+\|v\|_{2^{*}}^{2^{*}}\right) .
$$


Observe that $\|u\|_{2}^{2}+\|v\|_{2}^{2} \leq C_{2}\|(u, v)\|_{\lambda}^{2}$, we have

$$
J_{\lambda}(u, v) \geq \frac{1}{4}\|(u, v)\|_{\lambda}^{2}-\lambda C_{\delta}\left(\|u\|_{2^{*}}^{2^{*}}+\|v\|_{2^{*}}^{2^{*}}\right),
$$

which implies that the conclusions required hold. The proof is completed.

Lemma 3.8. Under the assumptions of Theorem 3, for any finite dimensional subspace $F \subset E_{\lambda}$, we get

$$
J_{\lambda}(u, v) \rightarrow-\infty \text { as }(u, v) \in F,\|(u, v)\|_{\lambda} \rightarrow \infty .
$$

Proof. By the assumptions of Theorem 3, one has

$$
J_{\lambda}(u, v) \leq \frac{1}{2}\|(u, v)\|_{\lambda}^{2}-\lambda a_{0}\left(|u|_{p}^{p}+|v|_{q}^{q}\right) \quad \text { for all }(u, v) \in E_{\lambda} .
$$

Since all norms in a finite dimensional space are equivalent and $p, q>2$, we easily obtain the desired conclusion.

Define the functional

$$
\begin{aligned}
\Phi_{\lambda}(u, v)= & \frac{1}{2} \int_{\mathbb{R}^{N}}\left(|\nabla u+i \sqrt{\lambda} A u|^{2}+|\nabla v+i \sqrt{\lambda} B v|^{2}\right. \\
& \left.+\lambda V(x)\left(|u|^{2}+|v|^{2}\right)\right)-a_{0} \lambda \int_{\mathbb{R}^{N}}|u|^{p}+|v|^{q} .
\end{aligned}
$$

It is obvious that $\Phi_{\lambda} \in C^{1}\left(E_{\lambda}\right)$ and $J_{\lambda}(u, v) \leq \Phi_{\lambda}(u, v),(u, v) \in E_{\lambda}$.

Note that

$$
\inf \left\{\int_{\mathbb{R}^{N}}|\nabla \phi|^{2}: \phi \in C_{0}^{\infty}\left(\mathbb{R}^{N}, \mathbb{R}\right),\|\phi\|_{p}=1\right\}=0
$$

and

$$
\inf \left\{\int_{\mathbb{R}^{N}}|\nabla \psi|^{2}: \phi \in C_{0}^{\infty}\left(\mathbb{R}^{N}, \mathbb{R}\right),\|\psi\|_{q}=1\right\}=0 .
$$

For any $\delta>0$, there exist $\phi_{\delta}, \psi_{\delta} \in C_{0}^{\infty}\left(\mathbb{R}^{N}, \mathbb{R}\right)$, with $\left\|\phi_{\delta}\right\|_{p}=\left\|\psi_{\delta}\right\|_{q}=1$, and $\operatorname{supp} \phi_{\delta}, \operatorname{supp} \psi_{\delta} \subset B_{r_{\delta}}(0)$ such that $\left\|\nabla \phi_{\delta}\right\|_{2}^{2},\left\|\nabla \psi_{\delta}\right\|_{2}^{2}<\delta$.

Let $e_{\lambda}(x)=\left(\phi_{\delta}(\sqrt{\lambda} x), \psi_{\delta}(\sqrt{\lambda} x)\right)$, then, suppe $e_{\lambda} \subset B_{\lambda^{-\frac{1}{2}} r_{\delta}}(0)$.

For $t \geq 0$, we have

$$
\begin{aligned}
\Phi_{\lambda}\left(t e_{\lambda}\right) & =\frac{t^{2}}{2}\left\|e_{\lambda}\right\|_{\lambda}^{2}-a_{0} \lambda t^{p} \int_{\mathbb{R}^{N}}\left|\phi_{\delta}(\sqrt{\lambda} x)\right|^{p}-a_{0} \lambda t^{q} \int_{\mathbb{R}^{N}}\left|\psi_{\delta}(\sqrt{\lambda} x)\right|^{q} \\
& =\lambda^{1-\frac{N}{2}} I_{\lambda}\left(t \phi_{\delta}, t \psi_{\delta}\right),
\end{aligned}
$$

where

$$
\begin{aligned}
I_{\lambda}(u, v)= & \frac{1}{2} \int_{\mathbb{R}^{N}}\left(\left|\nabla_{A} u\right|^{2}+\left|\nabla_{A} v\right|^{2}+\left(\left|A\left(\lambda^{-\frac{1}{2}} x\right)\right|^{2}+V\left(\lambda^{-\frac{1}{2}} x\right)\right)\left(|u|^{2}+|v|^{2}\right)\right) \\
& -a_{0} \int_{\mathbb{R}^{N}}\left(|u|^{p}+|v|^{q}\right) .
\end{aligned}
$$


Obviously, we get

$$
\begin{aligned}
& \max _{t \geq 0} I_{\lambda}\left(t \phi_{\delta}, t \psi_{\delta}\right) \\
\leq & \frac{p-2}{2 p\left(p a_{0}\right)^{\frac{2}{p-2}}}\left\{\int_{\mathbb{R}^{N}}\left|\nabla_{A} \phi_{\delta}\right|^{2}+\left(\left|A\left(\lambda^{-\frac{1}{2}} x\right)\right|^{2}+V\left(\lambda^{-\frac{1}{2}} x\right)\right)\left|\phi_{\delta}\right|^{2}\right\} \\
& +\frac{q-2}{2 q\left(q a_{0}\right)^{\frac{2}{q-2}}}\left\{\int_{\mathbb{R}^{N}}\left|\nabla_{A} \psi_{\delta}\right|^{2}+\left(\left|A\left(\lambda^{-\frac{1}{2}} x\right)\right|^{2}+V\left(\lambda^{-\frac{1}{2}} x\right)\right)\left|\psi_{\delta}\right|^{2}\right\} .
\end{aligned}
$$

Recall that $A(0)=0, B(0)=0, V(0)=0, \operatorname{supp} \phi_{\delta}, \operatorname{supp} \psi_{\delta} \subset B_{r_{\delta}}(0)$. Therefore, there exists $\Lambda_{\delta}>0$ such that, for all $\lambda \geq \Lambda_{\sigma}$, we get

(3.4) $\max _{t \geq 0} J_{\lambda}\left(t \phi_{\delta}, t \psi_{\delta}\right) \leq\left(\frac{p-2}{2 p\left(p a_{0}\right)^{\frac{2}{p-2}}}(5 \delta)^{\frac{p}{p-2}}+\frac{q-2}{2 q\left(q a_{0}\right)^{\frac{2}{q-2}}}(5 \delta)^{\frac{q}{q-2}}\right) \lambda^{1-\frac{N}{2}}$.

Lemma 3.9. Under the assumptions of Theorem 3, for any $\sigma>0$, there exists $\Lambda_{\sigma}>0$ such that for each $\lambda \geq \Lambda_{\sigma}$, there is $\bar{e}_{\lambda} \in E_{\lambda}$ with $\left\|\bar{e}_{\lambda}\right\|_{\lambda}>\rho_{\lambda}, J_{\lambda}\left(\bar{e}_{\lambda}\right) \leq$ 0 and

$$
\max _{t \geq 0} J_{\lambda}\left(t \bar{e}_{\lambda}\right) \leq \sigma \lambda^{1-\frac{N}{2}}
$$

where $\rho_{\lambda}$ is defined form Lemma 3.7.

Proof. This proof is similar to Lemma 4.3 in [12], it can be easily obtained.

For any $m \in N$, we can choose $m$ functions $\phi_{\delta}^{i} \in C_{0}^{\infty}\left(\mathbb{R}^{N}\right)$ such that $\operatorname{supp} \phi_{\delta}^{i} \cap$ $\operatorname{supp} \phi_{\delta}^{j}=\emptyset, i \neq j,\left\|\phi_{\delta}^{i}\right\|_{p}=1$ and $\left\|\nabla \phi_{\delta}^{i}\right\|_{2}^{2}<\delta$. Similarly, one can also get $m$ functions $\psi_{\delta}^{i} \in C_{0}^{\infty}\left(\mathbb{R}^{N}\right)$ with $\operatorname{supp} \psi_{\delta}^{i} \cap \operatorname{supp} \psi_{\delta}^{j}=\emptyset, i \neq j,\left\|\psi_{\delta}^{i}\right\|_{q}=1$ and $\left\|\nabla \psi_{\delta}^{i}\right\|_{2}^{2}<\delta$. Let $r_{\delta}^{m}>0$ be such that $\operatorname{supp}\left(\phi_{\delta}^{i}, \psi_{\delta}^{i}\right) \subset B_{r_{\delta}^{m}}^{i}(0)$ for $i=$ $1,2, \ldots, m$.

Set $e_{\lambda}^{i}(x)=\left(\phi_{\delta}^{i}(\sqrt{\lambda} x), \psi_{\delta}^{i}(\sqrt{\lambda} x)\right)=\left(f_{\lambda}^{i}, g_{\lambda}^{i}\right), i=1,2, \ldots, m$, then, $\operatorname{supp} e_{\lambda}^{i}(x)$ $\subset B_{\lambda^{-\frac{1}{2}} r_{\delta}^{m}}(0)$.

Let $F_{\lambda \delta}^{m}=\operatorname{span}\left\{e_{\lambda}^{1}, e_{\lambda}^{2}, \ldots, e_{\lambda}^{m}\right\}$. For each

$$
(u, v)=\sum_{i=1}^{m} k_{i} e_{\lambda}^{i} \in F_{\lambda \delta}^{m}
$$

we get

$$
\begin{aligned}
\int_{\mathbb{R}^{N}}\left(\left|\nabla_{A} u\right|^{2}+\left|\nabla_{B} v\right|^{2}\right) & =\sum_{i=1}^{m}\left|k_{i}\right|^{2}\left(\int_{\mathbb{R}^{N}}\left|\nabla_{A} f_{\lambda}^{i}\right|^{2}+\int_{\mathbb{R}^{N}}\left|\nabla_{B} g_{\lambda}^{i}\right|^{2}\right), \\
\int_{\mathbb{R}^{N}} V(x)\left(|u|^{2}+|v|^{2}\right) & =\sum_{i=1}^{m}\left|k_{i}\right|^{2}\left(\int_{\mathbb{R}^{N}} V(x)\left|f_{\lambda}^{i}\right|^{2}+\int_{\mathbb{R}^{N}} V(x)\left|g_{\lambda}^{i}\right|^{2}\right), \\
\frac{1}{2^{*}} \int_{\mathbb{R}^{N}} K(x)\left(|u|^{2^{*}}+|v|^{2^{*}}\right) & =\frac{1}{2^{*}} \sum_{i=1}^{m}\left|k_{i}\right|^{2^{*}}\left(\int_{\mathbb{R}^{N}} K(x)\left|f_{\lambda}^{i}\right|^{2^{*}}+\int_{\mathbb{R}^{N}} K(x)\left|g_{\lambda}^{i}\right|^{2^{*}}\right)
\end{aligned}
$$


and

$$
\int_{\mathbb{R}^{N}} H(u, v)=\sum_{i=1}^{m} \int_{\mathbb{R}^{N}} H\left(k_{i} f_{\lambda}^{i}, k_{i} g_{\lambda}^{i}\right)
$$

Therefore

$$
J_{\lambda}(u, v)=\sum_{i=1}^{m} J_{\lambda}\left(k_{i} e_{\lambda}^{i}\right)
$$

and

$$
J_{\lambda}\left(k_{i} e_{\lambda}^{i}\right) \leq \phi_{\lambda}\left(k_{i} e_{\lambda}^{i}\right) .
$$

Set $\beta_{\delta}:=\max \left\{\left\|\left(\phi_{\delta}^{i}, \psi_{\delta}^{i}\right)\right\|_{2}^{2}: i=1,2, \ldots, m\right\}$ and choose some $A_{m \delta}>0$ such that

$$
V\left(\lambda^{\frac{-1}{2}} x\right) \leq \frac{\delta}{\beta_{\delta}} \quad \text { for all }|x| \leq r_{\delta}^{m} \text { and } \lambda \geq \Lambda_{m \delta}
$$

Similar to the proof mentioned above, we can acquire the following inequality

$$
\max _{(u, v) \in F_{\lambda \delta}^{m}} J_{\lambda}(u, v) \leq\left(\frac{m(p-2)}{2 p\left(p a_{0}\right)^{\frac{2}{p-2}}}(5 \delta)^{\frac{p}{p-2}}+\frac{m(q-2)}{2 q\left(q a_{0}\right)^{\frac{2}{q-2}}}(5 \delta)^{\frac{q}{q-2}}\right) \lambda^{\frac{2-N}{2}} .
$$

By using the estimate, we can get the following result.

Lemma 3.10. Under the assumptions of Lemma 3.7, for any $m \in \mathbb{N}$ and $\sigma>0$, there exists $\Lambda_{m \sigma}>0$ such that for each $\lambda \geq \Lambda_{m \delta}$, there exists a $m$ dimensional subspace $F$ satisfying

$$
\max _{(u, v) \in F} J_{\lambda}(u, v) \leq \sigma \lambda^{\frac{2-N}{2}} .
$$

Proof. Choose $\sigma>0$ so small that

$$
\left(\frac{m(p-2)}{2 p\left(p a_{0}\right)^{\frac{2}{p-2}}}(5 \delta)^{\frac{p}{p-2}}+\frac{m(q-2)}{2 q\left(q a_{0}\right)^{\frac{2}{q-2}}}(5 \delta)^{\frac{q}{q-2}}\right) \leq \sigma
$$

and take $F=F_{\lambda \delta}^{m}$. By (3.5), we get the conclusion as required.

\section{Proof of the main results}

Proof of Theorem 1. By Lemma 3.9, for any $0<\sigma<\alpha_{0}$, there exists $\Lambda_{\sigma}>0$, such that for each $\lambda \geq \Lambda_{\sigma}$, we have $c_{\lambda} \leq \sigma \lambda^{1-\frac{N}{2}}$, where

$$
\begin{aligned}
c_{\lambda} & =\inf _{\gamma \in \Gamma_{\lambda}} \max _{t \geq 0} J_{\lambda}(\gamma(t)), \\
\Gamma_{\lambda} & =\left\{\gamma \in C\left([0,1], E_{\lambda}\right): \gamma(0)=0, \gamma(1)=\bar{e}_{\lambda}\right\} .
\end{aligned}
$$

In virtue of Lemma $3.5, J_{\lambda}$ satisfies the $(P S)_{c_{\lambda}}$ condition. Hence, by the mountain pass theorem, there exists $\left(u_{\lambda}, v_{\lambda}\right) \in E_{\lambda}$ satisfying $J_{\lambda}^{\prime}\left(u_{\lambda}, v_{\lambda}\right)=$ 0 and $J_{\lambda}\left(u_{\lambda}, v_{\lambda}\right)=c_{\lambda}$. Therefore, $\left(u_{\lambda}, v_{\lambda}\right)$ is a weak solution of (2.1). Moreover, it is well known that $\left(u_{\lambda}, v_{\lambda}\right)$ is a least energy solution of (2.1).

Note that $J_{\lambda}\left(u_{\lambda}, v_{\lambda}\right) \leq \sigma \lambda^{1-\frac{N}{2}}, J_{\lambda}^{\prime}\left(u_{\lambda}, v_{\lambda}\right)=0$ and

$$
J_{\lambda}\left(u_{\lambda}, v_{\lambda}\right)=J_{\lambda}\left(u_{\lambda}, v_{\lambda}\right)-\frac{1}{\theta} J_{\lambda}^{\prime}\left(u_{\lambda}, v_{\lambda}\right)\left(u_{\lambda}, v_{\lambda}\right)
$$




$$
\begin{aligned}
= & \left(\frac{1}{2}-\frac{1}{\theta}\right)\left\|\left(u_{\lambda}, v_{\lambda}\right)\right\|_{\lambda}^{2}+\left(\frac{1}{\theta}-\frac{1}{2^{*}}\right) \lambda \int_{\mathbb{R}^{N}} K(x)\left(\left|u_{\lambda}\right|^{2^{*}}+\left|v_{\lambda}\right|^{2^{*}}\right) \\
& +\lambda \int_{\mathbb{R}^{N}}\left(\frac{1}{\theta}\left(u_{\lambda} H_{u}\left(u_{\lambda}, v_{\lambda}\right)+v_{\lambda} H_{v}\left(u_{\lambda}, v_{\lambda}\right)\right)-H\left(u_{\lambda}, v_{\lambda}\right)\right) \\
\geq & \left(\frac{1}{2}-\frac{1}{\theta}\right)\left\|\left(u_{\lambda}, v_{\lambda}\right)\right\|_{\lambda}^{2} .
\end{aligned}
$$

So, the diamagnetic inequality implies that

$$
\frac{\theta-2}{2 \theta} \int_{\mathbb{R}^{N}}\left(|\nabla| u_{\lambda}||^{2}+|\nabla| v_{\lambda}||^{2}+\lambda V(x)\left(\left|u_{\lambda}\right|^{2}+\left|v_{\lambda}\right|^{2}\right)\right) \leq \sigma \lambda^{1-\frac{N}{2}} .
$$

The proof is completed.

Proof of Theorem 2. By Lemma 3.10, for any $m \in \mathbb{N}$ and $\sigma \in\left(0, \alpha_{0}\right)$, there exists $\Lambda_{m \sigma}$ such that for $\lambda \geq \Lambda_{m \sigma}$, we can choose a $m$-dimensional subspace $F$ with $\max \Phi_{\lambda}(F) \leq \sigma \lambda^{1-\frac{N}{2}}$. By Lemma 3.8, there is $R>0$ (depending on $\lambda$ and $m$ ) such that $\Phi_{\lambda}(u) \leq 0$ for all $u \in F \mid B_{R}$.

Denote the set of all symmetric (in the sense that $-\Omega=\Omega$ ) and closed subsets of $E_{\lambda}$ by $\Sigma$. For each $\Omega \in \Sigma$, let $\operatorname{gen}(\Omega)$ be the Krasnoselski genus and set

$$
i(A):=\min _{h \in \Gamma_{m}}\left(h(\Omega) \cap \partial B_{\rho_{\lambda}}\right),
$$

where $\Gamma_{m}$ is the set of all odd homeomorphisms $h \in C\left(E_{\lambda}, E_{\lambda}\right)$ and $\rho_{\lambda}$ is the number of Lemma 3.7. Then $i$ is a version of Benci's pseudoindex [5]. Let

$$
c_{\lambda_{j}}=\inf _{i(\Omega) \geq j} \sup _{u \in \Omega} J_{\lambda}(u), 1 \leq j \leq m .
$$

Since $J_{\lambda}(u) \geq \alpha_{\lambda}$ for all $u \in \partial B_{\rho_{\lambda}}$ (see Lemma 3.7) and $i(F)=\operatorname{dim} F=m$, we have

$$
\alpha_{\lambda} \leq c_{\lambda_{1}} \leq c_{\lambda_{2}} \leq \cdots \leq c_{\lambda_{m}} \leq \sup _{(u, v) \in F_{\lambda \sigma}^{m}} J_{\lambda}(u, v) \leq \sigma \lambda^{1-\frac{N}{2}} .
$$

In connection with Lemma 3.6, we know that $J_{\lambda}$ satisfies the $(P S)_{c_{\lambda_{j}}}$ condition at all levels $c_{\lambda_{j}}$. By the critical point theory, all $c_{\lambda_{j}}$ are critical levels and $J_{\lambda}$ has at least $m$ pairs of non-trivial critical points. Finally, as in the proof of Theorem 1, we easily get these solutions are the least energy solutions.

Acknowledgements. The authors thank the anonymous referee for precious comments and suggestions about the original manuscript.

\section{References}

[1] A. Ambrosetti, M. Badiale, and S. Cingolani, Semiclassical states of nonlinear Schrödinger equations, Arch. Ration. Mech. Anal. 140 (1997), no. 3, 285-300.

[2] A. Ambrosetti, A. Malchiodi, and S. Secchi, Multiplicity results for some nonlinear Schrödinger equations with potentials, Arch. Ration. Mech. Anal. 159 (2001), no. 3, 253-271.

[3] G. Arioli and A. Szulkin, A semilinear Schrödinger equation in the presence of a magnetic field, Arch. Ration. Mech. Anal. 170 (2003), no. 4, 277-295. 
[4] T. Bartsch, E. N. Dancer, and S. Peng, On mult-bump semi-classical bound states of nonlinear Schrödinger equations with electromagnetic fields, Adv. Differential Equations 11 (2006), no. 7, 781-812.

[5] V. Benci, On critical point theory for indefinite functionals in the presence of symmetries, Trans. Amer. Math. Soc. 274 (1982), no. 2 533-572.

[6] D. Cao and Z. Tang, Existence and uniqueness of multi-bump bound states of nonlinear Schrödinger equations with electromagnetic fields, J. Differential Equations 222 (2006), no. 2, 381-424.

[7] S. Cingolani, Semiclassical stationary states of nonlinear Schrödinger equation with an external magnetic field, J. Differential Equations 188 (2003), no. 1, 52-79.

[8] S. Cingolani and M. Lazzo, Multiple positive solutions to nonlinear Schrödinger equation with competing potential functions, J. Differential Equations 160 (2000), no. 1, 118-138.

[9] S. Cingolani and M. Nolasco, Multi-peaks periodic semiclassical states for a class of nonlinear Schrödinger equation, Proc. Roy. Soc. Edinburgh Sect. A 128 (1998), no. 6, 1249-1260.

[10] S. Cingolani and S. Secchi, Semiclassical states for NLS equations with magnetic potentials having polynomial growths, J. Math. Phys. 46 (2005), no. 5, 053503, 19 pp.

[11] M. Clapp and Y. H. Ding, Minimal nodal solutions of a Schrödinger equation with critical nonlinearity and symmetric potential, Differential Integral Equations 16 (2003), no. 8, 981-992.

[12] Y. H. Ding and F. H. Lin, Solutions of perturbed Schrödinger equations with critical nonlinearity, Calc. Var. Partial Differential Equations 30 (2007), no. 2, 231-249.

[13] M. Esteban and P. L. Lions, Stationary solutions of nonlinear Schrödinger equation with an external magnetic field, in PDE and Calculus of Variations, in honor of E. De Giorgi, Brikhäuser, 1990, 369-408.

[14] A. Floer and A. Weinstein, Nonspreading wave packets for the cubic Schrödinger equation with a bounded potential, J. Funct. Anal. 69 (1986), no. 3, 397-408.

[15] Y. G. Oh, On positive multi-lump bound states of nonlinear Schrödinger equations under multiple well potential, Comm. Math. Phys. 131 (1990), no. 2, 223-253.

[16] M. del Pino and P. Felmer, Semi-classical states for nonlinear Schrödinger equations, J. Funct. Anal. 149 (1997), no. 1, 245-265.

[17] — Multi-peak bound states for nonlinear Schrödinger equations, Ann. Inst. H. Poincaré Anal. Non Lineaire 15 (1998), no. 2, 127-149.

[18] Z. Tang, On the least energy solutions of nonlinear Schrödinger equations with electromagnetic fields, Comput. Math. Appl. 54 (2007), no. 5, 627-637.

[19] _ Multi-bump bound states of nonlinear Schrödinger equations with electromagnetic fields and critical frequency, J. Differential Equations 245 (2008), no. 10, 27232748.

[20] F. Wang, On an electromagnetic Schrödinger equation with critical growth, Nonlinear Anal. 69 (2008), no. 11, 4088-4098.

[21] X. Wang, On concentration of positive bound states of nonlinear Schrödinger equations, Comm. Math. Phys. 153 (1993), no. 2, 229-244.

[22] M. Willem, Minimax Theorems, Birkhäuser, Boston, MA, 1996.

HuIXING ZHANG

Department of Mathematics

China University of Mining and Technology

Xuzhou, Jiangsu 221116, P. R. China

E-mail address: huixingzhangcumt@163.com 
WENBIN LiU

Department of Mathematics

China University of Mining and Technology

Xuzhou, Jiangsu 221116, P. R. China

E-mail address: wblium@163.com 